\title{
THE SPECTRUM OF THE OLD NOVA V603 AQL OBSERVED WITH THE HST
}

\author{
M. FRIEDJUNG ${ }^{1}$, P. L. SELVELLI ${ }^{2}$, A. CASSATELLA ${ }^{1}$ \\ 1. Institut d'Astrophysique (CNRS), 98 bis Boulevard Arago, \\ 75014 Paris, France \\ 2. Osservatorio Astronomico, Via G,B. Tiepolo 11, \\ 34131 Trieste, Italy \\ 3. Instituto Astrofisica Spaziale (CNR), Via E. Fermi 21, \\ 00044 Frascati, Italy
}

\begin{abstract}
Spectra of the old nova V603 Aql taken with the HST-GHRS did not reveal resonance doublet absorption components arising in material ejected in the 1918 outburst. However absorption components, probably produced by a disk wind, were observed, though most line emission probably comes from regions associated with an accretion disk. Most strikingly, dramatic line profile variations on time-scales of not much more than $10 \mathrm{~m}$ were seen.
\end{abstract}

\section{Introduction}

V603 Aql is a bright old nova, whose eruption was observed in 1918. Due to its brightness it appears a favorable target to search for lines produced by absorption in the ejected envelope as well as due to the disk wind. In the present work the regions of the $\mathrm{CIV}, \mathrm{N} \mathrm{V}$ and Si IV resonance doublets were observed in 1994 Febuary with the Goddard High Resolution Spectrograph of the HST at a resolution of $210^{4}$. Each exposure was programmed to be split into 4 sub-exposures of $11 \mathrm{~m}$ (C IV), $10.5 \mathrm{~m} \mathrm{(N} \mathrm{v)} \mathrm{and} 8 \mathrm{~m}$ (Si IV), in order to reduce fixed pattern noise of the diode array.

\section{The search for absorption lines due to the ejected envelope}

Absorption components might be expected at a blue-shift near -1900 $\mathrm{km} \mathrm{s}^{-1}$, corresponding to the blue-shift of the envelope ejected in 1918. None were seen on the summed spectra. If spherical symmetry of the enve-

329

A. Evans and J. H. Wood (eds.), Cataclysmic Variables and Related Objects, 329-330.

(C) 1996 Kluwer Academic Publishers. Printed in the Netherlands. 
lope is assumed, with $\mathrm{C}$ and $\mathrm{N}$ abundances and ionization fractions of recent novae following the consistent analysis of Andreä, Drechsel \& Starrfield (1994), we find a maximum envelope mass $\sim 210^{-5} \mathrm{M}_{\odot}$. The mass could be considerably larger if the envelope had broken up into dense clumps.

\section{Line profiles due to the central remnant}

Broad spectral features were however detected. These were blue-shifted absorption for the $\mathrm{N} v$ doublet, a $\mathrm{P}$ Cygni absorption and emission profile for C IV and mainly emission for Si IV. The summed emission profiles are not far from symmetric and, in the case of $\mathrm{C}$ IV, very strong compared with the blue-shifted absorption. Comparing them with what are perhaps the best calculated disk wind profiles of Knigge, Woods \& Drew (1995) for a probable orbital inclination of $17^{\circ}$ (Ritter 1990), the emission is far too strong. It is hard to explain such emission unless most came from regions near the disk in Keplerian orbits. The emission line widths are sufficiently large to indicate emission near the white dwarf, suggesting that at least most of the disk is not broken up by a magnetic field. On the other hand the absorption components and the disk emission are explainable as due to a disk wind.

The most remarkable result however is the variations in the line profiles between the sub-exposures. In particular the blue-shifted absorption components of $\mathrm{NV}$ and $\mathrm{CIV}$ even disappeared at times; CIV showed the strongest absorption on a sub-exposure following one with no absorption. The time-scale, not much more than $\sim 10 \mathrm{~m}$, is short compared with the orbital period of $199 \mathrm{~m}$. The simplest explanation is that of rapid variations in the state of ionization.

\section{References}

Andreä, J., Drechsel, H., Starrfield, S., 1994, A\&A, 291, 869

Knigge, C., Woods, J.A., Drew, J.E., 1995, MNRAS, 273, 225

Ritter, H., 1990, A\&AS, 85, 1179 